\title{
ELABORACIÓN DE UNA BEBIDA FUNCIONAL DE ALTO VALOR BIOLÓGICO A BASE DE BOROJO (Borojoa patinoi Cuatrec)
}

\section{FORMULATION OF A FUNCTIONAL BEVERAGE OF HIGH BIOLOGICAL VALUE BASED ON BOROJO (Borojoa patinoi Cuatrec)}

\author{
Guillermo Salamanca G., Mónica Patricia Osorio T., Leidy Marcela Montoya \\ Grupo de Investigaciones Mellitopalinológicas y Propiedades Fisicoquímicas de Alimentos, \\ Facultad de Ciencias Universidad del Tolima. Tolima, Colombia
}

\begin{abstract}
A functional food of high biological value from Borojó pulp using honey as a sweetener and supported in a yogurt base has been evaluated and optimized in 16 different formulations in the range of 5-15\% pulp; 7082.5\% of yogurt and 5 a 15\% of honey from iSabana de Bogotâi. Based on the ratings physicochemical and sensory analysis was found the optimum formulation with $12.5 \%$ of pulp, 75.0 base milk yogurt y $12.5 \% \mathrm{w} / \mathrm{w}$ honey. The sensory properties have important differences that contribute to the process of optimization. The stability of the product at $8^{\circ} \mathrm{C}$ is 30 days. The mixture showed no significant microbial flora due to safety of materials.
\end{abstract}

Key words: Borojoa patinoi Cuatrec.; borojo; functional foods; probiotics; prebiotics.

Este trabajo fue recibido el 20 de Mayo de 2009 y aceptado para ser publicado el 17 de Diciembre de 2009.

\section{INTRODUCCIÓN}

En el diseño de productos y procesos agroalimentarios, es frecuente acudir a las herramientas estadísticas de diseño robusto y experimental para la valoración y optimización de mezclas de varios componentes o ingredientes [1,2]. En la elaboración de jugos, néctares, conservas, compotas, mermeladas, cremogenados o yogures, se requiere encontrar la mezcla óptima de ingredientes que permita generar un nuevo producto cuya formulación ofrezca características de producto funcional con alto valor nutricional y en las que se mantengan propiedades organolépticas de aroma y sabor deseables $[3,4]$. En el desarrollo de nuevos productos generalmente se acude a los diseños mezclas, para optimizar las proporciones de las componentes. La forma como se analizan este tipo de diseño es a través de una superficie de respuesta, que es la que permite encontrar la formulación óptima de una serie de mezcla de prueba [5].

Los frutos de Borojoa patinoi, corresponden a una especie arbórea y endémica de la región biogeográficas del Chocó; es un árbol del sotobosque. Produce un fruto carnoso de alto valor nutricional que lo posicionan como un recurso promisorio [6,7]. Se usa para preparar conservas y vino, pero su principal uso es como bebida refrescante con posibilidades para su transformación usando tecnología de Spray-dryer [8]. La pulpa de este frutal presenta un alto contenido en fósforo, buen nivel de carbohidratos, vitaminas, aminoácidos y elementos básicos para la alimentación humana. Ha sido reconocido como fruto con propiedades $[9,10]$. El yogurt es un derivado lácteo asociado a procesos biotecnológicos selectivos en los cuales se usan agentes microbianos del género Lactobacillus, Bifidobacterium, Enterococcus o Pediococcus. Es un producto funcional, que actúa como defensa natural de los procesos digestivos, aporta vitaminas A, B y minerales, su consumo se ha incrementado de manera importante; usado como vehículo para la incorporación de nutrientes, fibra y distintos tipos de frutas que aportan antioxidantes incrementando su funcionalidad [11].

Los trabajos sobre desarrollo de productos funcionales con pulpas de Borojó, hasta ahora se comienzan a considerar. El objetivo de este trabajo ha sido elaborar un cremolácteo de fruta de borojó usando una base de 
yogurt y miel como fuente de glucosa y fructosa que le confieren el valor energizante en una sola matriz optimizada mediante diseños experimentales de mezclas.

\section{MATERIALES Y MÉTODOS}

Materia prima: El estudio se realizó sobre pasta comercial de Borojó, adquirida en un supermercado de cadena de la ciudad de Ibagué (Tolima-Colombia); como edulcorante se uso miel beneficiada en apiarios del sector de la Sabana (Cundinamarca-Colombia) y como base láctea se uso yogur generado de un proceso estandarizado en la planta de lacteos del Servicio Nacional de Aprendizaje SENA La granja Espinal.

Caracterizaciones: Se estimaron las propiedades fisicoquímicas de las muestras de pulpa de Borojó, miel y base de yogur. Los parámetros considerados en la caracterización de la miel se hicieron siguiendo métodos armonizados de la comisión Internacional de la miel [12]. Adicionalmente se realizaron valoraciones polínicas y evaluaciones microbiológicas para aerobios mesófilos, enterobacterias, mohos y levaduras [13]. Así como pruebas sensoriales cuantitativas descriptivas en cada uno de los productos y formulaciones de las mezclas estudiadas usando metodologías de análisis cuantitativo descriptivo, QDA [14,15]. La composición de la pulpa fue determinada mediante análisis proximal y determi- naciones específicas siguiendo metodología descrita en el manual de métodos analíticos para alimentos de la AOAC [16]. Las determinaciones de las propiedades térmicas de la pulpa de Borojó se estimaron a partir del análisis proximal a través de relaciones funcionales descritas por Salamanca [12].

Determinación de fenoles: Sobre la pulpa de fruta se determinó el contenido de fenoles totales por maceración con $10 \mathrm{ml}$ de metanol acuoso (80:20), para extraer los fenoles libres sobre una base de $5 \mathrm{~g}$ de muestra por $24 \mathrm{~h}$. Luego se realizaron separaciones por centrifugaron a $4000 \mathrm{rpm}$. Sobre los sólidos generados se procedió con una segunda extracción con la misma cantidad de solución metanólica. Las fracciones resultantes se mezclaron y se analizaron por espectrofotometría UV-Visible a $765 \mathrm{~nm}$ usando el reactivo de Folin-Ciocalteau, con preparación previa de la curva de calibración usando ácido gálico como patrón [16].

Diseño experimental: Las variables experimentales estudiadas fueron proporciones de pulpa, yogurt y miel, 16 tratamientos se definieron usando un diseño de mezclas empleando el paquete Minitab $15^{\mathrm{TM}}$. Las formulaciones fueron preparadas utilizando diferentes concentraciones de pulpa de fruta: 5-15\%; yogurt 70$85 \%$ y miel tipo sabana $5-15 \%$, las fracciones de los componentes se representan en la figura 1.

\section{FIGURA 1}

Proporciones de pulpa de fruta, yogurt y miel en fracciones propuestas durante el proceso de optimización de la bebida funcional a base de Borojó

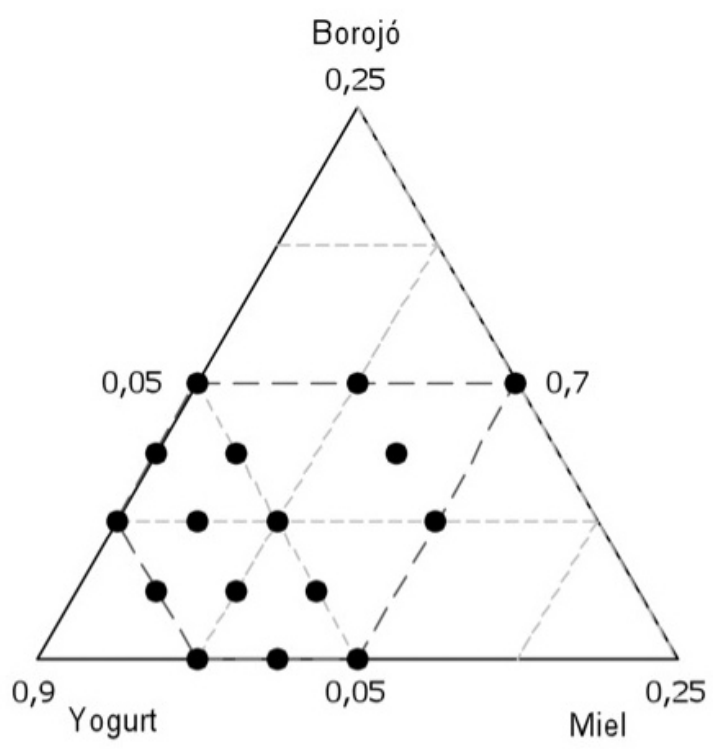


Análisis fisicoquímico: Las variables de respuesta analizadas fueron $\mathrm{pH}$, acidez titulable, conductividad, sólidos iónicos solubles totales y ${ }^{\circ} \mathrm{Brix}$, determinadas de acuerdo a metodologías de la AOAC [15]; las propiedades cromáticas (CIElab), se establecieron mediante registros fotográficos continuos siguiendo la metodología descrita por León [17].

Evaluación sensorial: Se realizaron pruebas sensoriales a través de una escala hedónica para cada una de las formulaciones de la base láctea y pulpa de Borojó. La escala técnica es como se indica: 0-2, malo; 3-4, aceptable; 5-6, bueno y 7-9, excelente, sobre los parámetros de aroma, sabor, viscosidad y aceptación global; participaron 16 panelistas entrenados, a quienes se les presentaron cada una de las formulaciones. Las evaluaciones se hicieron en periodos diferentes para evitar saturación y cansancio de los jueces, debido al elevado número de muestras a analizar. En fase de selección de catadores y de manera previa se realizaron pruebas de selección de panelistas aplicando el software Quali-Sense 1.0@. Los resultados globales de las formulaciones se analizaron siguiendo la metodología del
Análisis Cuantitativo Descriptivo QDA. Los parámetros evaluados fueron: aceptación, apariencia, aroma, color, consistencia, sabor global, sabor residual a Borojó y textura bucal.

Análisis microbiológico: En la determinación de aerobios mesófilos y enterobacterias, fue usado el método de recuento estándar en placa por siembra profunda, con incubación a $35^{\circ} \mathrm{C}$ por 48 horas. El recuento de mohos y levaduras se realizó en agar PDA con inclusión de cloranfenicol a $22^{\circ} \mathrm{C}$ por 5 días [12].

Modelos de optimización: En el estudio de modelos en los que participan variables respuesta, durante la optimización de los componentes en una formulación, se generan aproximaciones que representen las relaciones funcionales. El modelo evaluado correspondió a un sistema de segundo orden de la forma:

$$
Y=\beta_{0}+\sum_{i=1}^{3} \beta_{i} X_{i}+\sum_{i=1(j<1)}^{3} \sum_{j=1}^{3} \beta_{i j} X_{i} X_{j}+\sum_{i=1}^{3} \beta \beta_{i i} X_{i}^{2}
$$

Donde Y es la respuesta evaluada; los términos $\beta 0$, es el intercepto; $\beta \mathrm{ij} \beta \mathrm{ii}$ son constantes y representan

\begin{tabular}{llc}
\multicolumn{3}{c}{ TABLA 1 } \\
\multicolumn{3}{c}{ Composición media de la pulpa de Borojó } \\
\multicolumn{1}{c}{ Parámetro } & Unidades & \\
Humedad & $\% \mathrm{p} / \mathrm{p}$ & Valores \\
Proteínas & $\% \mathrm{p} / \mathrm{p}$ & $64.8 \pm 0.5$ \\
Carbohidratos & $\% \mathrm{p} / \mathrm{p}$ & $0.71 \pm 0.03$ \\
Fibra & $\% \mathrm{p} / \mathrm{p}$ & $37.1 \pm 1.10$ \\
Cenizas & $\% \mathrm{p} / \mathrm{p}$ & $10.2 \pm 0.92$ \\
Fibra & $\% \mathrm{p} / \mathrm{p}$ & $0.77 \pm 0.02$ \\
Grasa & $\% \mathrm{p} / \mathrm{p}$ & $10.2 \pm 1.11$ \\
Calcio & $\mathrm{mg} / 100 \mathrm{~g}$ & $0.83 \pm 0.12$ \\
Fósforo & $\mathrm{mg} / 100 \mathrm{~g}$ & 26.0 \\
Hierro & $\mathrm{mg} / 100 \mathrm{~g}$ & 160 \\
Tiamina & $\mathrm{mg} / 100 \mathrm{~g}$ & 1.61 \\
Riboflavina & $\mathrm{mg} / 100 \mathrm{~g}$ & 0.30 \\
Niacina & $\mathrm{mg} / 100 \mathrm{~g}$ & 0.12 \\
Vitamina C & $\mathrm{mg} / 100 \mathrm{~g}$ & 2.30 \\
Ascórbico & $\mathrm{mg} / 100 \mathrm{~g}$ & 3.10 \\
pH & $\mathrm{Unidades}$ & 3.00 \\
Actividad de agua & $\mathrm{aw}$ & 2.72 \\
Capacidad calorífica $(\mathrm{Cp})$ & $\mathrm{Jkg}-1^{\circ} \mathrm{C}-1$ & 0.978 \\
Conductividad térmica $(\mathrm{k})$ & $\mathrm{Wm}-1^{\circ} \mathrm{C}-1$ & 3391 \\
Difusividad térmica $\mathrm{x} 10-7(\alpha)$ & $\mathrm{m} 2 \mathrm{~s}-1$ & 0.501 \\
Entalpia $\left(20^{\circ} \mathrm{C}\right)$ & $\mathrm{J} \mathrm{kg}-1$ & 1.35 \\
Calorías & $\mathrm{kcal}$ & 383580 \\
& & 0.090 \\
\hline
\end{tabular}


los coeficientes de ajuste de la regresión cuadrática; las variables $\mathrm{Xi} \mathrm{Xj}$, son interacciones de primer orden entre $\mathrm{Xi}$ y $\mathrm{Xj}$ para $(\mathrm{j}<1)[5]$.

\section{RESULTADOS Y DISCUSIÓN}

Pulpa de borojó: El fruto presenta un peso medio de $0.70 \mathrm{Kg}$, constituido por un $88 \%$ de pulpa, $12 \%$ de semillas; su pulpa es de color café; los parámetros de cromaticidad corresponden a una luminancia de 6.65 (L), 3.58 (a*: rojo/verde) y 4.83 (b*: amarillo/azul) y 6.2 para el croma (C). La pulpa es de carácter ácido $(\mathrm{pH}$ 2.7), humedad de $64.8 \pm 0.5 \%$, el contenido proteínico es de $0.70 \pm 0.03$; fibra dietaría $22.7 \pm 1.10 \%$ y $2.67 \pm 0.12 \%$ de pectina. Los carbohidratos totales son del orden de $37 \%$, con presencia mayoritaria de fructosa, glucosa y sacarosa.

La pulpa alberga $7.20 \%$ p/p de fenoles (ácido gálico), que le confieren potencial antioxidante. Los resultados del análisis proximal, se recogen en la tabla 1. La densidad del material es de $1095.5\left(\mathrm{~kg} / \mathrm{m}^{3}\right)$. A pesar de ser una fruta poco perecedera y de alta disponibilidad en su medio natural, el borojó es un producto de difícil manejo debido a su consistencia. Las propiedades térmicas de la pulpa están representadas en una capacidad calorífica de $3391.4(\mathrm{~J} / \mathrm{kg} \mathrm{K})$; conductividad y difusividad térmica de $0.501\left(\mathrm{~W} / \mathrm{m}^{\circ} \mathrm{K}\right)$ y $1.35 \times 10^{-7}(\mathrm{~m} 2 / \mathrm{s})$ respectivamente.

Miel: Las propiedades fisicoquímicas observadas en las muestras de miel, son propias de una tipo sabana con $19 \%$ de humedad, índice de refracción de 1.487; fracción en azúcares fructosa (38.7), glucosa (31.4); acidez total es de 28.4 (meq/Kg), con un $\mathrm{pH}$ de 3.66; conductividad de $3.00 \times 10-4(\mu \mathrm{S} / \mathrm{cm}) ; 0.140 \%$ para cenizas, 0.597 actividad de agua e índice de Tabouret superior a 1.70. Las calorías estimadas son del orden de $0.310 \mathrm{Kcal} / 100 \mathrm{~g}$. El aroma de la miel es intenso y fino, ligeramente acaramelado, presenta baja y alta viscosidad. Su calidad microbiológica es notable, no presentan coliformes y el recuento para aeróbios mesófilos a $31^{\circ} \mathrm{C}$ es inferior a 250(ufc/g), con reducidos niveles en mohos y levaduras <100(ufc/g).

Yogur: La composición de la base láctea utilizada, presenta una humedad de $80.4 \%$; proteína $3.5 \%$, fibra bruta 0.10 y cenizas $0.82 \% \mathrm{p} / \mathrm{p}$; el aporte energético es equivalente a $12.5 \mathrm{Kcal}$. La acidez total es de $1.12 \%$ (p/p como ácido láctico). En la tabla $2 \mathrm{~A}$, se indica la composición media de la base láctea de yogur.

Análisis de mezclas: En el diseño de mezclas, los factores que intervienen en la formulación son las proporciones de las componentes y las respuestas a optimizar son función de éstas con respecto al total y no dependen de la cantidad de cada componente. Una característica especial de los diseños de mezclas es que la cantidad total de la mezcla normalmente se fija en el diseño y la de cada componentes es proporcional a la total, además la proporción de los componentes no varía de manera independientemente, como si ocurre en los diseños factoriales, que están restringidas a que la suma sea constante (1 ó 100\%). En la formulación para la elaboración de la bebida láctea y funcional usando borojo y miel, ésta última actúa como edulcorante natural, provee

TABLA 2 A

\section{Composición del media del yogur}

\begin{tabular}{llc}
\multicolumn{1}{c}{ Parámetro } & Unidades & Valores \\
Humedad & $\% \mathrm{p} / \mathrm{p}$ & $80.4 \pm 2.10$ \\
Proteínas & $\% \mathrm{p} / \mathrm{p}$ & $3.50 \pm 0.86$ \\
Carbohidratos & $\% \mathrm{p} / \mathrm{p}$ & $7.30 \pm 1.12$ \\
Fibra & $\% \mathrm{p} / \mathrm{p}$ & $12.7 \pm 1.31$ \\
Cenizas & $\% \mathrm{p} / \mathrm{p}$ & $0.12 \pm 0.02$ \\
Fibra & $\% \mathrm{p} / \mathrm{p}$ & $0.10 \pm 0.02$ \\
Grasa & $\% \mathrm{p} / \mathrm{p}$ & $0.77 \pm 0.03$ \\
Calcio & $\mathrm{mg} / 100 \mathrm{~g}$ & 182 \\
Fósforo & $\mathrm{mg} / 100 \mathrm{~g}$ & 157 \\
Hierro & $\mathrm{mg} / 100 \mathrm{~g}$ & 0.12 \\
Vitamina A & $\mu \mathrm{g} / 100 \mathrm{~g}$ & 9.0 \\
Vitamina C & $\mathrm{mg} / 100 \mathrm{~g}$ & 0.98 \\
pH & $\mathrm{Unidades}$ & 4.12 \\
Contenido energético & $\mathrm{Kcal}$ & 12.5
\end{tabular}




\section{FIGURA 2}

Perfil del aporte de electrolitos de los componentes a las formulaciones de la bebida funcional

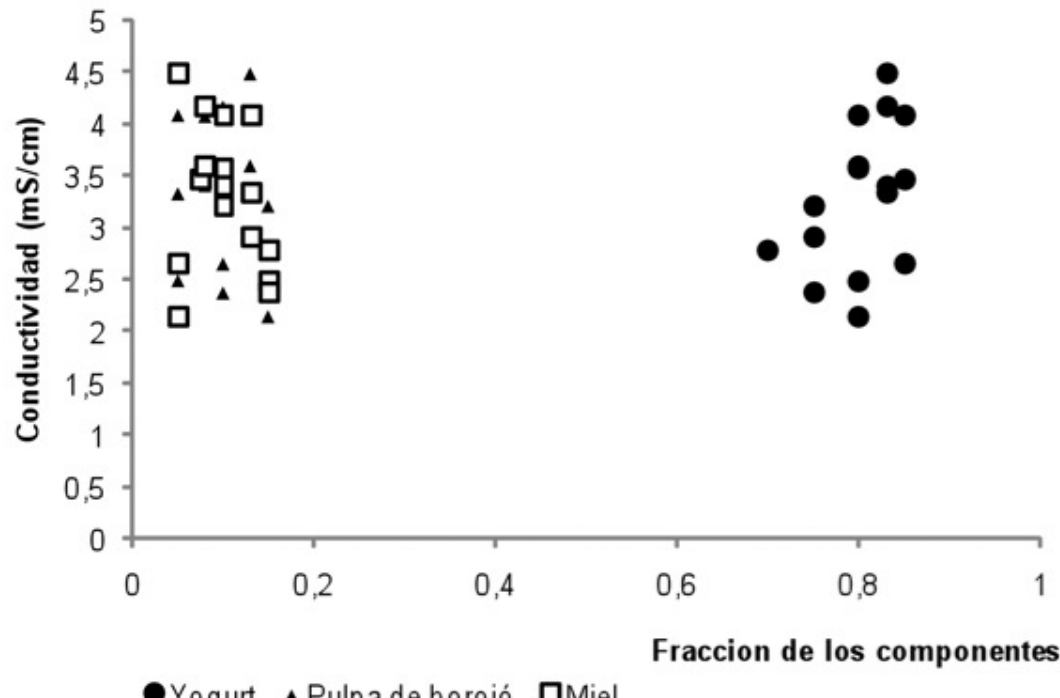

\section{TABLA 2 B}

Parámetros fisicoquímicos asociados a las formulaciones de la bebida funcional a base de borojó

\begin{tabular}{ccccccccc} 
Formulación & $\begin{array}{c}\text { Pulpa de } \\
\text { borojo }\end{array}$ & $\begin{array}{c}\text { Base de } \\
\text { yogur }\end{array}$ & Miel & ${ }^{\mathbf{}}$ Brix & $\begin{array}{c}\text { Acidez } \\
(\mathbf{\%} \text { láctico } \mathbf{p} / \mathbf{p})\end{array}$ & $\begin{array}{c}\mathbf{p H} \\
\left(\mathbf{2 5} \mathbf{0}^{\mathbf{C}}\right)\end{array}$ & $\begin{array}{c}\text { Conductividad } \\
\mathbf{m S} / \mathbf{c m}\end{array}$ & $\begin{array}{c}\text { Fenoles } \\
(\mathbf{m g} / \mathbf{K g ~ A . ~ G a ́ l i c o ) ~}\end{array}$ \\
\hline 1 & 0.15 & 0.80 & 0.05 & 18 & 1.50 & 3.78 & 2.15 & 108 \\
2 & 0.05 & 0.80 & 0.15 & 22 & 0.88 & 4.05 & 2.50 & 36.0 \\
3 & 0.15 & 0.70 & 0.15 & 23 & 0.71 & 3.72 & 2.80 & 108 \\
4 & 0.10 & 0.85 & 0.05 & 17 & 1.30 & 3,87 & 2.66 & 72.0 \\
5 & 0.05 & 0.85 & 0.10 & 19 & 1.11 & 4.00 & 4.10 & 36.0 \\
6 & 0.05 & 0.83 & 0.13 & 20 & 1.12 & 4.01 & 3.34 & 36.0 \\
7 & 0.13 & 0.83 & 0.05 & 16 & 1.38 & 3.73 & 4.50 & 93.6 \\
8 & 0.15 & 0.75 & 0.10 & 20 & 1.45 & 3.62 & 3.22 & 108 \\
9 & 0.08 & 0.85 & 0.08 & 17 & 1.24 & 3.89 & 3.48 & 57.6 \\
10 & 0.10 & 0.75 & 0.15 & 24 & 1.77 & 3.74 & 2.38 & 72.0 \\
11 & 0.10 & 0.80 & 0.10 & 19 & 1.30 & 3.78 & 3.58 & 72.1 \\
12 & 0.13 & 0.80 & 0.08 & 18 & 1.43 & 3.69 & 3.61 & 93.6 \\
13 & 0.08 & 0.80 & 0.13 & 18 & 1.23 & 3.85 & 4.09 & 57.6 \\
14 & 0.13 & 0.75 & 0.13 & 19 & 1.34 & 3.68 & 2.92 & 93.6 \\
15 & 0.10 & 0.83 & 0.08 & 18 & 1.40 & 3.78 & 4.18 & 72.0 \\
16 & 0.08 & 0.83 & 0.10 & 20 & 1.25 & 3.85 & 3.42 & 57.7
\end{tabular}


los azúcares simples glucosa y fructosa que contribuyen a las propiedades palatables de las mezclas generadas.

El aporte energético de los componentes estimados a partir del análisis proximal, indica que la pulpa de fruta aporta $0.09 \mathrm{Kcal} / 100$; la miel 0.31 y la base láctea 85.0 $\mathrm{Kcal} / 100 \mathrm{~g}$. En las formulaciones finales el estimado energético es de $10 \pm 1 \mathrm{Kcal} / 100 \mathrm{~g}$ de bebida (39 a 44 $\mathrm{kJ} / 100 \mathrm{~g}$ ). Teniendo como referencia los requerimientos nutricionales y los valores guía en calorías de las raciones dietéticas diarias recomendadas (RDDR) por edades [18], el consumo de dos raciones de $250 \mathrm{~g}$ o una de 500 , contribuye con $3.35 \pm 0.45 \%$ del total de los requerimientos diarios en niños, $1.74 \pm 0.23$ en adultos, $1.95 \pm 0.26$ en varones, $2.43 \pm 0.32$ en mujeres y 2.21 $\pm 0.30 \%$ en embarazadas.

Las mezclas evaluadas, presentan carácter ácido (pH 3.70 a 4.01), con una acidez total (\% p/p ac. láctico) entre 0.70 a 1.77 ; la conductividad se incrementa en la medida en que aumentan las proporciones de los componentes en las formulaciones; éstas van desde 2.0 a 4.5 $\mathrm{mS} / \mathrm{cm}$. El aporte de electrolitos se hace desde la base láctea dado que en las mezclas finales éste componente está en mayor proporción, el carácter ácido del yogur contribuye a la conductividad del edulcorante y la pulpa de fruta adicionada (figura 2).

Caracterización de formulaciones: Los parámetros fisicoquímicos de las mezclas presentan diferencias en el contenido de humedad desde 51 a $61 \%$; el pH fluctúa entre 5,00 y 5.98 a $25^{\circ} \mathrm{C}$. Las propiedades cromáticas L (luminosidad), a* (rojo/verde), b* (amarillo/azul), C (croma) y h (ángulo de tono), varían ligeramente dentro de rangos estrechos. Con $10 \%$ de fruta, $75 \%$ de base láctea y $15 \%$ de miel, se logra un contenido de $24{ }^{\circ}$ Brix. El contenido de antioxidantes como ácido gálico está en función de la pulpa con $36 \mathrm{mg} / \mathrm{Kg}$, cuando las proporciones de fruta, base láctea y miel son $5 \%, 85 \%$ y $10 \%$ en su orden y de $108 \mathrm{mg} / \mathrm{Kg}$ para las proporciones 15:80:5 (tabla 2 B); los parámetros cromáticos en las muestras evaluadas presentan variaciones principalmente sobre la cromaticidad rojo/verde (a*), amarillo/azul (b*) y el croma (C) tabla 3.

Los coeficientes asociados a los parámetros fisicoquímicos de mezclas generadas se presentan en la tabla 4. En todos los casos se presentan interacciones entre los componentes como aporte a la variabilidad de los parámetros, pero sin una mayor significancia estadística $(\mathrm{Pv}>0.05)$; las principales significancias estadísticas se observaron en el caso de la acidez en la interacción Borojo*Yogur (Pv 0.002) así como en el pH para borojo* yogurt, borojo* miel y yogurt *miel. La varianza explicada en cada uno de los modelos es mayor en el caso del pH y los sólidos solubles. Las figuras 3 a 5, muestran los promedios asociados a la significancia de los parámetros de acidez, pH y sólidos solubles.

\section{TABLA 3}

Parámetros cromáticos observados en las formulaciones de una bebida funcional a base de Borojó

\begin{tabular}{cccccccc} 
Formulación & $\begin{array}{c}\text { Pulpa de } \\
\text { borojo }\end{array}$ & $\begin{array}{c}\text { Base de } \\
\text { Yogur }\end{array}$ & $\mathbf{L}$ & $\mathbf{a}^{*}$ & $\mathbf{b}^{*}$ & $\mathbf{C}$ & $\mathbf{h}$ \\
\hline 1 & 0.15 & 0.80 & 55.8 & 1.27 & 12.2 & 12.3 & 84.1 \\
2 & 0.05 & 0.80 & 58.5 & -1.24 & 10.9 & 11.0 & 83.6 \\
3 & 0.15 & 0.70 & 53.7 & 0.22 & 14.7 & 14.78 & 89.1 \\
4 & 0.10 & 0.85 & 59.3 & -1.20 & 8.51 & 8.60 & 81.9 \\
5 & 0.05 & 0.85 & 61.2 & -0.71 & 8.20 & 8.2 & 85.0 \\
6 & 0.05 & 0.83 & 60.4 & -1.14 & 10.5 & 10.6 & 83.3 \\
7 & 0.13 & 0.83 & 52.8 & 1.27 & 13.3 & 13.3 & 84.5 \\
8 & 0.15 & 0.75 & 54.2 & 1.61 & 13.5 & 13.6 & 83.2 \\
9 & 0.08 & 0.85 & 56.7 & 0.87 & 11.4 & 11.5 & 85.6 \\
10 & 0.10 & 0.75 & 54.5 & -0.81 & 12.8 & 12.8 & 87.0 \\
11 & 0.10 & 0.80 & 58.3 & 0.80 & 10.5 & 10.5 & 85.6 \\
12 & 0.13 & 0.80 & 55.3 & 0.30 & 11.5 & 11.5 & 88.5 \\
13 & 0.08 & 0.80 & 61.1 & -2.12 & 11.3 & 11.5 & 79.3 \\
14 & 0.13 & 0.75 & 57.3 & -0.82 & 11.6 & 11.6 & 85.9 \\
15 & 0.10 & 0.83 & 57.7 & 0.63 & 10.6 & 10.7 & 86.6 \\
16 & 0.08 & 0.83 & 58.2 & -1.55 & 12.5 & 12.6 & 82.9
\end{tabular}


Valoraciones microbiológicas: La flora microbiana de los óptimos del diseño en las formulaciones, respecto de los aerobios mesófilos fue $<102$ ufc/g; para mohos y levaduras $<10 \mathrm{ufc/g}$ y ausencia de coliformes fecales. No existe diferencia significativa dentro de las valoraciones realizadas a todas las formulaciones de cremolácteos de borojó.

Evaluación sensorial: El análisis sensorial no revela diferencia significativas dentro las muestras con relación al aroma, observándose aceptabilidad por parte de los jueces; el modelo que las explica es lineal $(\mathrm{Pv}<0.05)$; En cuanto al dulzor y acidez existe incon-

\begin{tabular}{|c|c|c|c|c|c|c|c|c|c|}
\hline & Coef & $\begin{array}{r}\text { entes asoc } \\
\text { de p }\end{array}$ & $\begin{array}{l}\text { idos a l } \\
\text { pa de f }\end{array}$ & $\begin{array}{l}\text { TABLA } \\
\text { parámetr } \\
\text { ita de bor }\end{array}$ & $\begin{array}{l}\text { S fisico } \\
\text { o, miel }\end{array}$ & $\begin{array}{l}\text { micos } \\
\text { ogur }\end{array}$ & nezclas & & \\
\hline Coeficiente & ${ }^{\circ}$ Brix & Acidez & pH & Conduc. & $\mathbf{L}$ & $\mathbf{a}^{*}$ & $\mathbf{b}^{*}$ & $\mathbf{C}$ & $\mathbf{h}$ \\
\hline$\beta_{1}$ & 151 & -100 & 32.1 & -62.4 & 124 & 203 & 56.7 & 59.5 & 115 \\
\hline$\beta_{2}$ & 18.5 & -2.00 & 4.95 & 2.40 & 59.5 & -0.10 & 5.30 & 5.10 & 93.3 \\
\hline$\beta_{3}$ & 361 & -37.3 & 28.2 & -229 & -375 & -143 & 86.7 & 74.4 & 181 \\
\hline$\beta_{12}$ & -164 & 141 & -39.4 & 64.8 & -144 & -230 & -6.60 & -8.70 & 102 \\
\hline$\beta_{13}$ & -468 & 88.2 & -63.8 & 414 & 356 & -66.9 & -168 & -167 & 480 \\
\hline$\beta_{23}$ & -369 & 58.5 & -30.8 & 270 & 539 & 162 & -61.8 & -44.5 & 202 \\
\hline $\mathrm{R}^{2}, \%$ & 80.2 & 58.3 & 96.4 & 42.3 & 65.7 & 58.8 & 57.7 & 56.8 & 28.8 \\
\hline Ajust. R²,\% & 70.2 & 37.4 & 94.7 & 13.4 & 48.6 & 38.3 & 36.4 & 35.3 & - \\
\hline
\end{tabular}

\section{FIGURA 3}

Representación de los principales efectos sobre el pH, en las formulaciones de la bebida funcional

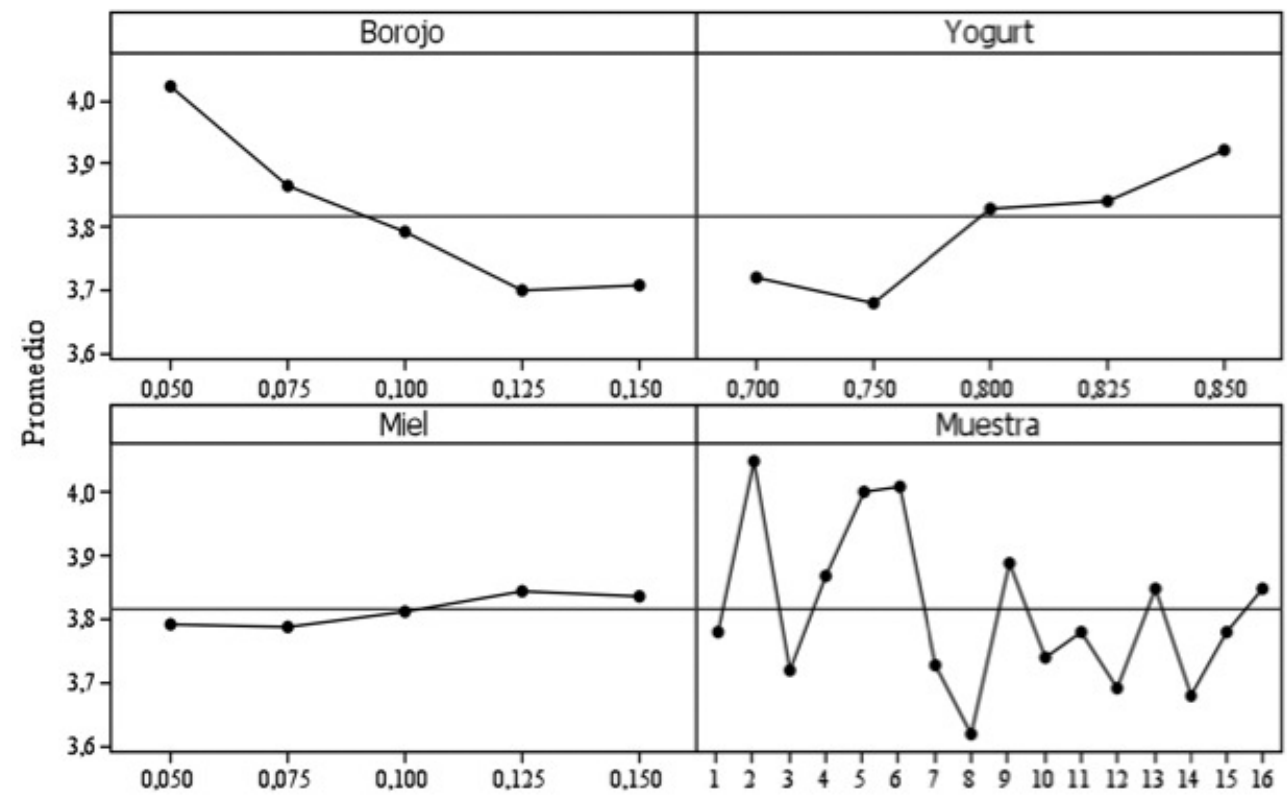




\section{FIGURA 4}

Representación de los principales efectos sobre la acidez en las formulaciones

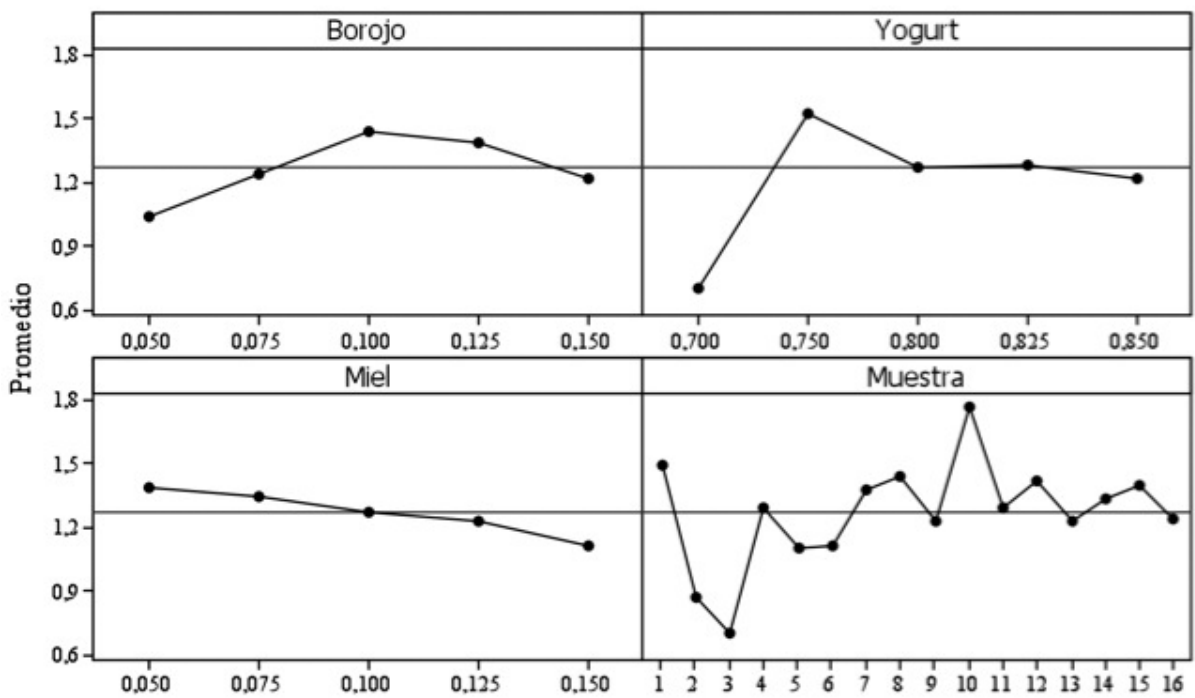

FIGURA 5

Representación de los principales efectos sobre los sólidos solubles

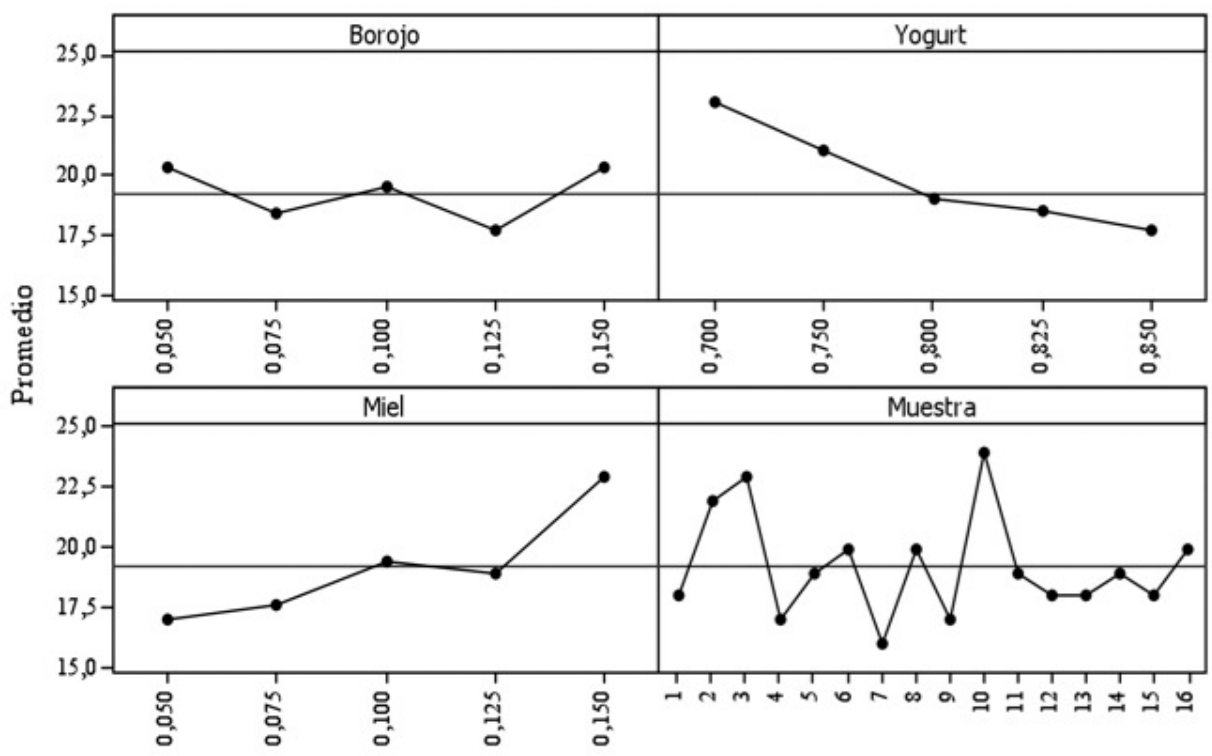


formidad, principalmente, por muestras que poseen niveles bajos de miel y altos de fruta. La sensación del color y la palatabidad se ajustan a modelos cuadráticos significativos $(\mathrm{Pv}<0.05)$, la luminancia $\mathrm{a}^{*} \mathrm{y} \mathrm{b} *$ a modelos lineales. Las mezclas con mayor aceptación son aquellas que presentan niveles elevados de borojó y miel, permitiendo percibir un equilibrio dulzor-ácido-palatabilidad. En la figura 6, se muestra en una escala hedónica de 0 a 9 los valores medios de la formulación mejor valoradas en el panel sensorial.

Optimización: Teniendo en cuenta la influencia de los parámetros con aporte significativo dentro de las variables experimentales y de acuerdo a la región óptima de aceptación sensorial de las mezclas (figura 3), las proporciones indicadas de los componentes corresponden a $12.5 \%$ de borojó, 12.5 de miel y $75 \%$ de yogurt.

\section{CONCLUSIONES}

El trabajo permitió desarrollar y optimizar una nueva forma de consumo de borojó (Borojoa patinoi Cuatrec.), en un cremolácteo endulzado con miel, a través de herramientas de diseño por superficie de respuesta. El producto optimizado mantiene las propiedades de la fruta, provee antioxidantes, minerales y vitaminas, aportados por sus componentes; la adición de miel re- salta los sabores y el aroma de la mezcla final; el valor calórico y los componentes en general del producto final lo clasifican como un alimento energético y funcional, que aporta calorías y puede ser consumido por un amplio grupo de personas. El aroma y flavor proveen al producto aceptabilidad sensorial, que a su vez está es influenciada por las variables ${ }^{\circ}$ Brix y $\mathrm{pH}$. La respuesta sensorial optimizada corresponde a una mezcla sobre la cual no revela actividad microbiológica importante. Dada la presencia de fenoles en la matriz de la fruta y el efecto de las bifidobacterias, el producto generado se presenta con un importante acierto que puede ser proyectado a un estudio de mercado.

\section{RESUMEN}

Se ha evaluado y optimizado un cremolacteo funcional y de alto valor biológico a partir de pulpa de Borojó usando miel como edulcorante y soportados en una base láctea de yogurt. Se evaluaron 16 formulaciones distintas en el rango $5-15 \%$ de pulpa; 70-82.5 de yogurt y 5 a $15 \%$ p/p de miel de la "Sabana de Bogotá". A partir de las valoraciones sensoriales y análisis fisicoquímicos se ha encontrado la formulación óptima con $12.5 \%$ de pulpa, 75.0 de base láctea de yogurt y $12.5 \%$ p/p de miel. Las propiedades sensoriales presentan diferencias importan-

\section{FIGURA 6}

Valores medios de la valoración sensorial de la bebida funcional

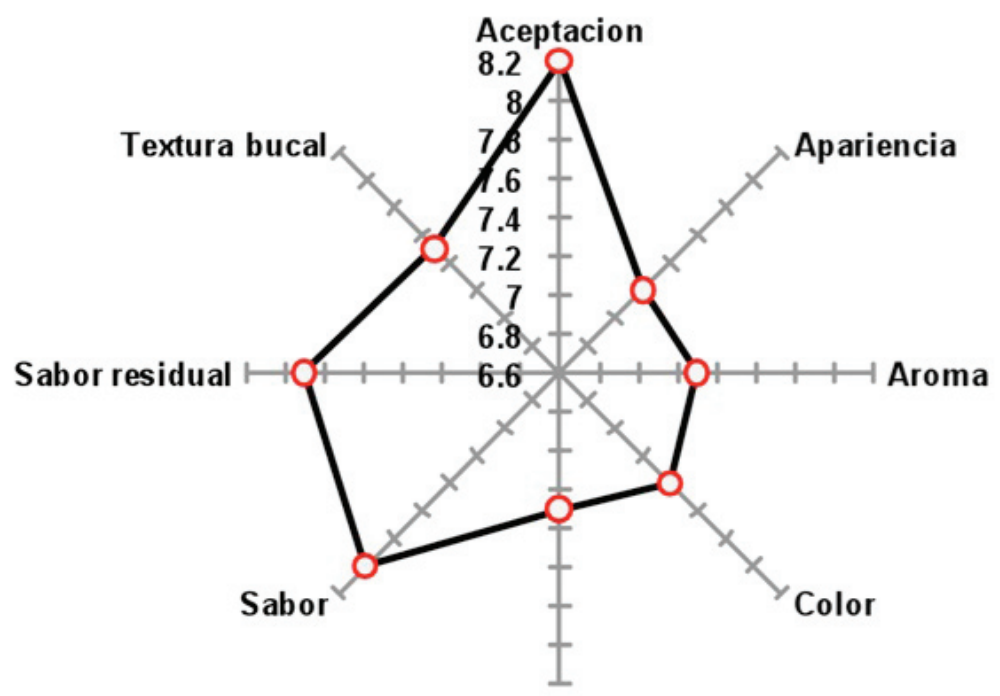

Consistencia 
tes, que contribuyen al proceso de la optimización. La estabilidad del producto a $8^{\circ} \mathrm{C}$ es de 30 días. La mezcla no revela flora microbiana importante y los niveles observados hacen del producto un material seguro.

Palabras clave: Borojoa patinoi Cuatre; borojó; alimentos funcionales; prebióticos; probióticos.

Dirigir la correspondencia a:

Profesor

Guillermo Salamanca G.

Barrio Santa Helena parte alta.

Ibagué Tolima Colombia A.A 546

salamancagrosso@gmail.com

\section{BIBLIOGRAFIA}

1. Voinovich D, Campisi B, Phan-Tan-Luu R. Experimental Design for Mixture Studies. Comprehensive Chemometrics, 2009, Chapter 1.13. pp 391-452.

2. Zhou Jian-zhong, LIU Xiao-li, Huang Kai-hong, Dong, Ming-sheng, Jiang Han-hu. Application of the Mixture Design to Design the Formulation of Pure Cultures in Tibetan kefir. Agricultural Sciences China 2007; (6): 11: 1383-1389.

3. Muteki K, MacGregor J F, Ueda T. Mixture designs and models for the simultaneous selection of ingredients and their ratios. Chemometrics Intelligent Laboratory Systems 2007; (86), 1: 17-25.

4. Aravind Mannarswamy A, Munson-McGee S H, Andersen P K. D-optimal designs for the Cross viscosity model applied to guar gum mixtures.

5. Álvarez H R, Salamanca G, Valoración metodológica para el estudio de mezclas ternarias en sistemas alimentarios Alimentos Ciencia Ingeniería 2007; 16: 92 - 96.

6. Angulo, M L., Salamanca G. La zona del Bajo Calima en el pacifico colombiano y su entorno frutícola. En Propiedades fisicoquímicas y sistemas de procesado: productos hortofrutícolas en el desarrollo agroalimentario. I Seminario Hortofrutícola Colombiano. I Congreso Iberoamericano sobre sistemas de procesado. Colciencias. Colciencias-Universidad del Tolima. Colombia. 2006: pp 14-17.

7. Giraldo C. I, Rengifo, L, Aguilar, E. Gaviria, D. Alegría, A H. Determinación del sexo en borojó (Borojoa patinoi, Cuatrecasas) mediante marcadores moleculares. Rev Colombiana Biotecnol. 2004: 2: 9-14.
8. Mosquera, L H, Moraga, G. Martínez-Navarrete, N. Effect of maltodextrin on the stability of freezedried borojó (Borojoa patinoi Cuatrec.) powder. J Food Engineering 2010: 9; 1; 72-78.

9. Mosquera, L. H., Rios, H. A. Potencialidades del borojó (Borojoa patinoi Cuatr.) en polvo como ingrediente natural. Propiedades Fisicoquímicas y Sistemas de Procesado: Productor Hortofrutícolas en el Desarrollo Agroalimentario. I Seminario Hortofrutícola Colombiano. I Congreso Iberoamericano sobre sistemas de procesado. Ibagué Tolima, Colombia. 2006: pp.182.

10. Mosquera, MH, Ríos, H. A..Zapata, P. S. Obtención de una materia prima con valor agregado mediante secado por aspersión a partir del fruto fresco de borojó (Borojoa patinoi Cuatr.). En: Rev Institucional Universidad Tecnológica Chocó. 2006: 11; $23 ; 5-10$.

11. Bogdanov S. Harmonised methods of the international honey commission. Swiss Bee Research Centre FAM, Liebefeld. Bern, Switzerland. International Honey Commission. 2002: pp.1- 61.

12. Salamanca, G Estudio analítico comparativo de las propiedades fisicoquímicas de mieles de Apis mellifera, en algunas zonas apícolas de los departamentos de Boyacá y Tolima (Colombia). Anales de las tesis doctorales fruto de la cooperación interuniversitaria con Colombia. Ed. Editorial de la Universidad Politécnica de Valencia. 1994-2005, 2005: I; 145-158.

13. Armstrong G A. Quantitative Descriptive Analysis (QDA) - utilising the human instrument. J Nutr Food Science 1999: 99 (6); 317-323.

14. Montenegro G, Gómez, P R., Casaubon, G, Peña, R. Implementación de un panel sensorial para mieles chilenas. Cien Inv Agr 2008: 35; 1; 51-58.

15. AOAC. Official Methods of Analysis, 17th edition. Association of official Anallytical Chemists, Washington, D.C. 2000.

16. Vinson, J A, Hao Y, Su X, Zubik L. Phenol Antioxidant Quantity and Quality in Foods: Vegetables J Agric Food Chem 1998: 46; 9; 3630-3634.

17. León K., Domingo M., Pedreschi F. Color meassurement in $\mathrm{La}^{*} \mathrm{~b} *$ units from RGB digital images. Food Res Internat 2006: 39; 10; 1084-1091.

18. Padilla, LE, Osorio, ML, En Bioquímica de J. J. Hicks. MacGraw-Hill. Interamericana. Capitulo 41. Nutrición Humana. 2001: 793 - 834 